\title{
Impaired WNT signaling and the spine-Heterozygous WNT1 mutation causes severe age-related spinal pathology
}

\section{Mäkitie, Riikka E.}

2017-08

Mäkitie , R E , Niinimaki , T , Nieminen , M T, Schalin-Jantti , C , Niinimaki , J \& Makitie , O 2017 , ' Impaired WNT signaling and the spine-Heterozygous WNT1 mutation causes severe age-related spinal pathology ' , Bone , vol. 101 , pp. 3-9 . https://doi.org/10.1016/j.bone.2017.04.001

http://hdl.handle.net/10138/237203

https://doi.org/10.1016/j.bone.2017.04.001

publishedVersion

Downloaded from Helda, University of Helsinki institutional repository.

This is an electronic reprint of the original article.

This reprint may differ from the original in pagination and typographic detail.

Please cite the original version. 
Full Length Article

\title{
Impaired WNT signaling and the spine-Heterozygous WNT1 mutation causes severe age-related spinal pathology
}

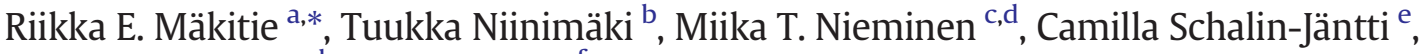 \\ Jaakko Niinimäki ${ }^{\text {c,d }}$, Outi Mäkitie ${ }^{\text {a,f,g }}$ \\ a Folkhälsan Institute of Genetics, University of Helsinki, Helsinki FI-00290, Finland \\ ${ }^{\mathrm{b}}$ Department of Orthopedics, Oulu University Hospital, Oulu FI-90220, Finland \\ ${ }^{c}$ Research Unit of Medical Imaging, Physics and Technology, Department of Diagnostic Radiology, Oulu University Hospital, Oulu FI-90220, Finland \\ ${ }^{d}$ Medical Research Center, University of Oulu and Oulu University Hospital, Oulu FI-90220, Finland \\ e Endocrinology, Abdominal Center, University of Helsinki and Helsinki University Hospital, Helsinki FI-00290, Finland \\ ${ }^{\mathrm{f}}$ Children's Hospital, University of Helsinki and Helsinki University Hospital, Helsinki FI-00290, Finland \\ ${ }^{g}$ Center for Molecular Medicine, Karolinska Institutet, and Clinical Genetics, Karolinska University Hospital, SE-171 76 Stockholm, Sweden
}

\section{A R T I C L E I N F O}

\section{Article history:}

Received 18 January 2017

Revised 24 February 2017

Accepted 6 April 2017

Available online 11 April 2017

\section{Keywords:}

WNT signaling

Magnetic resonance imaging

Vertebral compression fracture

Thoracic kyphosis

Schmorl node

Intervertebral disc

\begin{abstract}
A B S T R A C T
Background: WNT signaling plays a major role in bone and cartilage metabolism. Impaired WNT/B-catenin signaling leads to early-onset osteoporosis, but specific features in bone and other tissues remain inadequately characterized. We have identified two large Finnish families with early-onset osteoporosis due to a heterozygous WNT1 mutation c.652T $>$ G, p.C218G. This study evaluated the impact of impaired WNT/ß-catenin signaling on spinal structures.

Methods: Altogether 18 WNT1 mutation-positive (age range 11-76 years, median 49 years) and 14 mutationnegative subjects (10-77 years, median 43 years) underwent magnetic resonance imaging (MRI) of the spine. The images were reviewed for spinal alignment, vertebral compression fractures, intervertebral disc changes and possible endplate deterioration. The findings were correlated with clinical data.

Results: Vertebral compression fractures were present in 78\% (7/9) of those aged over 50 years but were not seen in younger mutation-positive subjects. All those with fractures had several severely compressed vertebrae. Altogether spinal compression fractures were present in 39\% of those with a WNT1 mutation. Only 14\% (2/14) mutation-negative subjects had one mild compressed vertebra each. The mutation-positive subjects had a higher mean spinal deformity index $(4.0 \pm 7.3$ vs $0.0 \pm 0.4)$ and more often increased thoracic kyphosis $(Z$-score $>$ +2.0 in 33\% vs 0\%). Further, they had more often Schmorl nodes (61\% vs 36\%), already in adolescence, and their intervertebral discs were enlarged.

Conclusion: Compromised WNT signaling introduces severe and progressive changes to the spinal structures. Schmorl nodes are prevalent even at an early age and increased thoracic kyphosis and compression fractures become evident after the age of 50 years. Therapies targeting the WNT pathway may be an effective way to prevent spinal pathology not only in those harboring a mutation but also in the general population with similar pathology.
\end{abstract}

(c) 2017 Elsevier Inc. All rights reserved.

\section{Introduction}

The importance of WNT/ $\beta$-catenin signaling for bone mass development was first discovered when mutations in the co-receptor, low-density lipoprotein receptor-related protein 5 (LRP5) were identified as the

* Corresponding author at: Folkhälsan Institute of Genetics, PO Box 63, University of Helsinki, Helsinki FI-00290, Finland.

E-mail addresses: riikka.makitie@helsinki.fi (R.E. Mäkitie), tuukka.niinimaki@oulu.fi (T. Niinimäki), miika.nieminen@oulu.fi (M.T. Nieminen), camilla.schalin-jantti@hus.fi (C. Schalin-Jäntti), jaakko.niinimaki@oulu.fi (J. Niinimäki), outi.makitie@helsinki.fi (O. Mäkitie). cause for the osteoporosis-pseudoglioma syndrome [1-2]. Since then, other skeletal disorders, including early-onset osteoporosis and various high bone mass disorders such as van Buchem disease and sclerosteosis due to either decreased or increased WNT signaling, respectively, have further underlined this pathway's significance in bone homeostasis [3-6]. Furthermore, several genome-wide association studies (GWAS) have identified LRP5 and other components of the WNT signaling pathway as major determinants of bone mass and fracture risk [7-8].

WNT signaling plays a major role in bone metabolism throughout life-in skeletal development during embryonic phase, in bone mass accrual during childhood and adolescence, and in maintenance of bone homeostasis and renewal in adulthood [9]. In adult bone, the WNT 
pathway stimulates bone formation by committing common mesenchymal osteochondroprogenitor cells towards the osteoblastic lineage, by promoting their proliferation and differentiation and by preventing the apoptosis of uncommitted progenitor cells and mature osteoblasts [10-11]. WNT signaling also indirectly both stimulates and represses osteoclastogenesis through osteoblast-derived receptor activator of nuclear factor-kappaB ligand (RANKL) and osteoprotegerin (OPG), respectively [12-13]. These mechanisms have been elucidated in several genetically modified mouse models, in which impaired WNT signaling leads to defects in bone formation and an osteoporotic phenotype [14-15]. Concurrently, WNT signaling also mediates chondrogenesis, sustains mature cartilage homeostasis in adults and, when improperly activated, leads to cartilaginous tissue pathology [16-17].

WNT1 has been identified as a key ligand for the WNT/ $\beta$-catenin pathway in bone, as several loss-of-function mutations in WNT1 were found to cause variable degrees of early-onset osteoporosis $[15,18,19]$. Apart from VCFs of the thoracic spine seen in plain radiographs [15], little is known about the effects of defective WNT signaling in the axial skeleton. Access to a unique research cohort with a large number of individuals with WNT1 osteoporosis prompted us to examine in detail the consequences of impaired WNT signaling in the axial skeleton with magnetic resonance imaging (MRI)-specifically, the prevalence and characteristics of VCFs, the possible changes in vertebral endplates and intervertebral discs, and the relationship of spinal changes with age. Our findings on 18 mutation-positive subjects indicate that impaired WNT signaling due to mutated WNT1 causes significant and progressive changes in the spine.

\section{Subjects and methods}

\subsection{Subjects}

During the course of our research program on early-onset osteoporosis, we identified two large Finnish families with autosomal dominant WNT1 osteoporosis. Initially, the first family included ten mutation-positive family members (Family A) [15]. All first-degree relatives at risk were subsequently offered genetic screening for the family's WNT1 mutation c.652T $>$ G (p.C218G). Through genetic screening of an additional 21 family members, 10 other subjects were found to harbor the mutation. The whole WNT1 gene was Sanger sequenced also for altogether $>250$ other Finnish index subjects with early-onset osteoporosis and another, seemingly unrelated family (Family B) was then found where four out of eight subjects were positive for the same WNT1 mutation as Family A. Thus altogether 24 WNT1 mutation-positive subjects were identified in these two families, both of ethnic Finnish background. In addition, 21 family members in these two families were confirmed by genetic screening to be negative for the mutation.

We offered all mutation-positive subjects from Families $A(n=20)$ and $\mathrm{B}(\mathrm{n}=4)$ an opportunity to participate in studies exploring the skeletal and extra-skeletal consequences of the heterozygous WNT1 mutation, including MRI evaluation of the spine. In order to be able to compare the findings with normal data, a control group with similar age and sex distribution was formed by offering participation to WNT1 mutation-negative individuals in families $A(n=17)$ and $B(n=2)$, aiming at similar age and sex distribution in the mutation-positive and control groups. Altogether 18 mutation-positive and 14 mutationnegative individuals consented. All subjects gave a written informed consent upon participation in the study. The research protocol was approved by the Research Ethics Board of Helsinki University Hospital.

\subsection{Genetic evaluations}

We performed genetic validations on DNA extracted from peripheral blood, as described previously [20]. We screened all samples for the families' known WNT1 heterozygous missense mutation c.652T $>$ G
(p.C218G) in exon 4 of the WNT1 gene (NCBI Reference Sequence NM_005430.3).

\subsection{Clinical cohort characteristics}

Mutation-positive subjects were clinically evaluated at Helsinki University Hospital for clinical features, including anthropometry, alignment and abnormalities of spine. For all subjects, data on sustained fractures and previous medical and surgical treatments were collected by interview and from medical records. History of back pain was recorded by interview; pain that required long-term medication, absence from work or professional help (e.g. physiotherapy) was considered disabling. All secondary causes of skeletal fragility (e.g. long-term illness or glucocorticoid treatment, low vitamin D status, or other biochemical or endocrine abnormality) had been ruled out previously $[15,20]$.

\subsection{Magnetic resonance imaging}

Magnetic resonance imaging (MRI) of the thoracic and lumbar spine were performed in 2015-2016 at the Oulu University Hospital with a 1.5-T scanner (MAGNETOM Aera; Siemens Healthcare, Erlangen, Germany) using a 32-channel spine coil. The imaging sequences included T1-weighted turbo inversion recovery magnitude (TIRM) sagittal images (TR/TE/TI 1900/10/860 ms, FOV $280 \times 280 \mathrm{~mm}$, matrix $320 \times 240$ pixels, slice thickness $4 \mathrm{~mm}$, and $0.8 \mathrm{~mm}$ interslice gap) and T2-weighted turbo spin echo (TSE) sagittal images (TR/TE 4000/ $99 \mathrm{~ms}$, FOV $280 \times 280 \mathrm{~mm}$, matrix $448 \times 358$ pixels, slice thickness $4 \mathrm{~mm}$, and $0.8 \mathrm{~mm}$ interslice gap) of lumbar spine and T2-weighted TSE sagittal images (TR/TE $3500 / 97 \mathrm{~ms}$, FOV $280 \times 280 \mathrm{~mm}$, matrix $384 \times 269$ pixels, slice thickness $4 \mathrm{~mm}$, and $0.8 \mathrm{~mm}$ interslice gap) of thoracic spine. All images were obtained in supine position with the subject lying down, legs extended horizontally.

The MRIs were first independently assessed for spinal changes by an orthopedic surgeon and an experienced radiologist who were blinded to the subjects' genotype and phenotype. The final conclusions were evaluated for discrepancy by calculating Intraclass Correlation Coefficient (ICC) for 10 randomly selected cases; for all the cases the ICC score was $>0.80$ and therefore the analyses were considered highly consistent and reproducible. The MRI data were assessed as described later.

\subsubsection{Vertebrae and intervertebral discs}

Changes were evaluated separately for each vertebral body. Thoracic and lumbar vertebrae were first classified according to the Genant et al. semi-quantitative method by grading each vertebral body and VCF from T4 to L4 as 0) normal; 1 ) mild (decrease in height 20-25\%); 2 ) moderate (decrease in height $25-40 \%$ ); or 3 ) severe (decrease in height $>40 \%$ ) [21]. The extent of vertebral changes was further evaluated using the spinal deformity index (SDI; scale 0-39) described by Minne et al. [22]. The SDI classification integrates number and severity of VCFs by summing all compression fracture scores. This classification has been proven accurate in assessing VCFs in osteoporotic patients [23].

\subsubsection{Endplates}

The number, location and extent of Schmorl nodes (SN) were recorded by using modified classification introduced by Samartzis et al. [24] The recorded domains were: vertebrae level (T4-L4), endplate involvement (rostral, caudal, or both) and size $(<1 / 3,<2 / 3$, or $>2 / 3$ endplate). The vertebrae having moderate or severe VCF (SDI $>1$ ) were not included in assessment.

\subsubsection{Spinal alignment}

Changes in spinal curvature were evaluated by calculating degrees for thoracic kyphosis and lumbar lordosis. Values were measured by selecting upper and lower vertebral bodies: T1 and T12 for thoracic spine, and L1 and L5 for lumbar spine. The angle was calculated using a modified Cobb's method by drawing tangent lines to these vertebral 
bodies; median values were used to compare differences between the two groups [25]. Measured values were also transformed into Z-scores based on age and sex-specific normative data according to Fon et al. [26].

\subsubsection{Statistical analysis}

Descriptive data are reported as median and range or as mean $\pm S D$. Normality of the data was assessed using Kolmogorov-Smirnov and Shapiro-Wilk and visually using histograms. Unpaired 2-tailed Student $t$ - and Mann-Whitney $U$ test were used as appropriate (SPSS Statistics 21; IBM Corporation, Armonk, NY, USA). A P-value $<0.050$ was considered statistically significant.

\section{Results}

\subsection{Cohort characteristics}

\subsubsection{Mutation-positive subjects}

The 18 WNT1 mutation-positive subjects (13 females, 5 males) ranged in age from 11 to 76 years (median 49 years) (Fig. 1, Table 1 ). Altogether $16(89 \%)$ of them had sustained at least one previous fracture. Twelve subjects (67\%) reported back pain; six of them considered the pain disabling. Five adults reported height loss of $\geq 4 \mathrm{~cm}$. None of the subjects reported high-impact trauma that would have caused spinal fractures. Nine subjects had received osteoporosis medication before the diagnosis of WNT1 osteoporosis and prior to the study and for three subjects, the treatment was still ongoing (Table 1).

\subsubsection{Mutation-negative subjects}

The 14 WNT1 mutation-negative subjects ( 7 females and males) ranged in age from 10 to 77 years (median 43 years) (Fig. 1, Table 1 ). Altogether $3(21 \%)$ of them had sustained one or more previous peripheral fractures, none had been diagnosed with compression fractures, three had mild back pain and one adult reported a $4 \mathrm{~cm}$ height loss. None had received any osteoporosis medications.

\subsection{MRI findings}

\subsubsection{Vertebrae}

VCFs were frequent and increasingly prevalent with age in the mutation-positive subjects; none of the subjects younger than 50 years had VCFs but seven out of nine (78\%) of those over 50 years had multiple VFCs ranging from mild to severe (Fig. 2A). In comparison, two out of the five mutation-negative subjects over the age of 50 years had a VCF, only one Genant grade 1 fracture each. The mean SDI-score for the mutation-positive subjects was $4 \pm 7.3$ and for the mutation-negative subjects $0 \pm 0.4(\mathrm{p}=0.07$ ) (Figs. 2 and $3 \mathrm{~A}-\mathrm{C})$. In the mutation-positive subjects, VCFs were more prevalent in the thoracic spine and either mild (Genant grade 1 ) or severe (Genant grade 3 ) while moderate changes (Genant grade 2) were present mainly in the lumbar spine.

\subsubsection{Vertebral endplates}

In two mutation-positive subjects, severe VCFs prevented the evaluation of SNs. Eleven (69\%) of the remaining 16 mutation-positive subjects had SN. In all of them SNs were present in the thoracic vertebrae and in six subjects, also in the lumbar spine (Figs. 3A and 4). Median number of SN in the mutation-positive subjects was 3 (range 0-16). In all these 11 subjects, the SNs were multilevel (i.e. seen in more than one endplate), present in all vertebral endplates, predominantly situated in T7 to $\mathrm{L} 1$ and most common in T9 (15\%). Altogether $57 \%$ of the SNs were rostral, $63 \%$ involved one third of an endplate, and none involved more than two thirds of an endplate. In mutation-negative subjects the SNs were less prevalent $(\mathrm{p}=0.07)$ as $5(36 \%)$ of the 14 subjects had SN; four in the thoracic spine, one in the lumbar spine and one subject in both. Median number of $\mathrm{SN}$ was 0 (range $0-3$ ). They were mostly rostral (64\%), most common in T11 (36\%) and mostly (64\%) one third of an endplate in size.

\subsubsection{Intervertebral discs}

Intervertebral discs in the lumbar spine appeared enlarged in the mutation-positive group (Fig. 3D). Median surface area of the intervertebral discs from L3 to L5 tended to be greater in the mutation-positive than in the mutation-negative subjects but this difference was not statistically significant ( $962 \mathrm{vs} 844 \mathrm{~mm}^{2}, \mathrm{p}=0.24$ ). Enlarged discs were biconcave in shape ("fish" or "hourglass vertebrae"). No enlarged discs were observed in the thoracic spine.

\subsubsection{Spinal alignment}

VCFs caused changes in the WNT1 subjects' spinal alignment. Thoracic kyphosis was significantly increased in the mutation-positive subjects and the median value for kyphosis was $39^{\circ}$ (vs $34^{\circ}, \mathrm{p}=0.01$ ). The median values of kyphosis for the mutation-positive and mutation-negative subjects under the age of 50 years were $36^{\circ}$ in both $(p=0.49)$, and

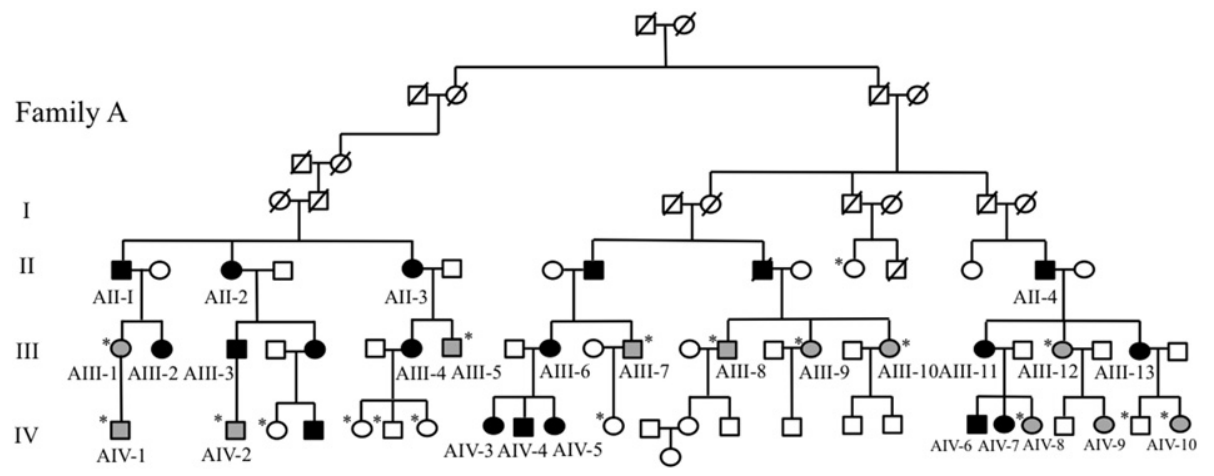

Family B

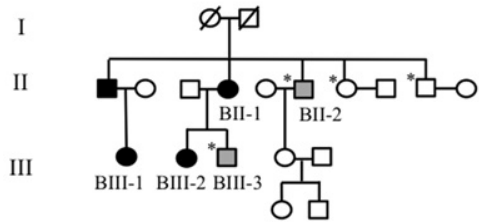

* Genetically screened for the mutation

- Mutation positive O $\square$ Mutation negative O Mutation negative control

Fig. 1. Pedigrees of the two Finnish families with a heterozygous p.C218G WNT1 mutation. The pedigrees have been modified to ensure anonymity. 
Table 1

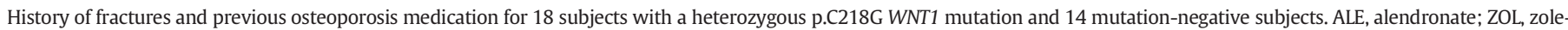
dronic acid; BP, bisphosphonate; O, denosumab; N/A, data not available; VCF, vertebral compression fracture.

\begin{tabular}{|c|c|c|c|c|c|c|c|c|}
\hline \multirow{2}{*}{ Subject } & \multirow{2}{*}{ Age (years) } & \multirow{2}{*}{$\operatorname{Sex}(M / F)$} & \multirow{2}{*}{ Osteoporosis medication } & \multicolumn{2}{|c|}{ Peripheral fractures } & \multirow{2}{*}{ History of VCF } & \multirow{2}{*}{ Back pain } & \multirow{2}{*}{ Height loss $(\mathrm{cm})$} \\
\hline & & & & Number & Age at 1 st fracture (years) & & & \\
\hline \multicolumn{9}{|c|}{ Mutation-positive subjects } \\
\hline AIV-6 & 11 & $\mathrm{~F}$ & None & 1 & 0 & - & - & 0 \\
\hline AIV-5 & 13 & M & None & 4 & $\mathrm{~N} / \mathrm{A}$ & - & - & 0 \\
\hline AIV-8 & 13 & $\mathrm{~F}$ & None & 1 & 8 & - & - & 0 \\
\hline AIV-4 & 17 & $\mathrm{~F}$ & None & 8 & 0 & - & - & 0 \\
\hline AIV-7 & 19 & M & None & 1 & 12 & - & - & 0 \\
\hline BIII-2 & 31 & $\mathrm{~F}$ & ALE 2008-2013 & 11 & 0.6 & - & + & 0 \\
\hline AIII-2 & 34 & $\mathrm{~F}$ & None & 2 & $\mathrm{~N} / \mathrm{A}$ & - & - & 0 \\
\hline AIII-12 & 44 & $\mathrm{~F}$ & None & 0 & - & - & + & 0 \\
\hline AIII-7 & 48 & $\mathrm{~F}$ & ZOL 2010- & 4 & $\mathrm{~N} / \mathrm{A}$ & - & + & 0 \\
\hline BII-1 & 51 & $\mathrm{~F}$ & $\mathrm{BP}$ (name N/A) 2002-2007 & 2 & 35 & - & + & $\mathrm{N} / \mathrm{A}$ \\
\hline AIII-14 & 52 & $\mathrm{~F}$ & None & 0 & - & - & + & $\mathrm{N} / \mathrm{A}$ \\
\hline AIII-3 & 52 & M & $\mathrm{N} / \mathrm{A}$ & 2 & 10 & + & + & 0 \\
\hline AIII-5 & 53 & $\mathrm{~F}$ & $\begin{array}{l}\text { RIS 2001-2003 } \\
\text { PTH 2012-2013 }\end{array}$ & 0 & - & + & + & 4 \\
\hline AII-1 & 63 & M & $\begin{array}{l}\text { ZOL 2007-2010 } \\
\text { PTH 2011-2013 }\end{array}$ & 3 & 18 & + & + & 7 \\
\hline BII-2 & 68 & $\mathrm{~F}$ & ALE 2007-2014 & 5 & $\mathrm{~N} / \mathrm{A}$ & - & + & 2 \\
\hline AII-2 & 72 & $\mathrm{~F}$ & $\begin{array}{l}\text { Estrogen (name N/A) } \\
\text { RIS 2006- }\end{array}$ & 9 & 7 & + & + & 13 \\
\hline AII-3 & 74 & $\mathrm{~F}$ & $\begin{array}{l}\text { ALE 2001-2006 } \\
\text { PTH 2006-2008 } \\
\text { ZOL 2008-2011 } \\
\text { DENO 2011- }\end{array}$ & 6 & 42 & + & + & 17 \\
\hline AII-4 & 76 & M & $\begin{array}{l}\text { ZOL } 2008 \\
\text { PTH 2011-2012 }\end{array}$ & 1 & $\mathrm{~N} / \mathrm{A}$ & + & + & 4 \\
\hline \multicolumn{9}{|c|}{ Mutation-negative subjects } \\
\hline AIV-1 & 10 & M & None & 0 & - & - & - & 0 \\
\hline AIV-2 & 16 & M & None & 0 & - & - & - & 0 \\
\hline AIV-10 & 18 & $\mathrm{~F}$ & None & 0 & - & - & - & 0 \\
\hline AIV-8 & 21 & $\mathrm{~F}$ & None & 0 & - & - & - & 0 \\
\hline AIV-9 & 27 & $\mathrm{~F}$ & None & 0 & - & - & - & 0 \\
\hline AIII-1 & 37 & $\mathrm{~F}$ & None & 0 & - & - & - & 0 \\
\hline AIII-5 & 42 & M & None & 0 & - & - & - & 0 \\
\hline BIII-3 & 43 & M & None & 0 & - & - & - & 0 \\
\hline AIII-12 & 49 & $\mathrm{~F}$ & None & 0 & - & - & - & 0 \\
\hline AIII-7 & 53 & M & None & 0 & - & - & - & 0 \\
\hline AIII-10 & 56 & $\mathrm{~F}$ & None & 5 & 19 & - & + & 4 \\
\hline AIII-9 & 57 & $\mathrm{~F}$ & None & 0 & - & - & - & 0 \\
\hline AIII-8 & 59 & $\mathrm{M}$ & None & 5 & 23 & - & $+1-$ & 0 \\
\hline BII-2 & 77 & M & None & 1 & 48 & - & + & 0 \\
\hline
\end{tabular}

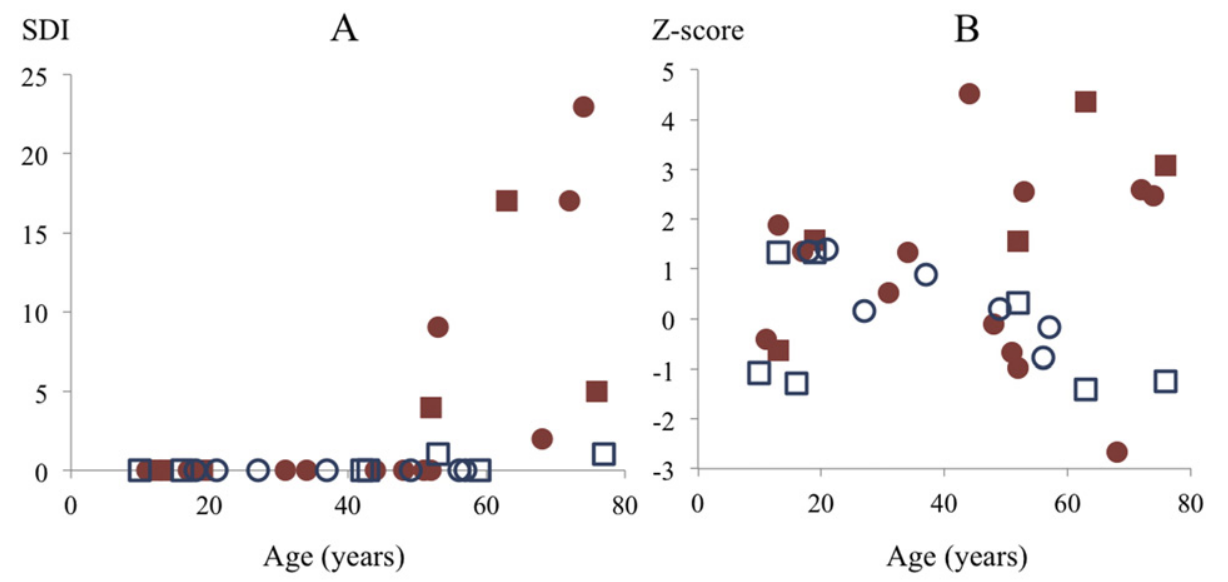

Mutation-positive, males

Mutation-positive, females

$\square$ Mutation-negative, males

O Mutation-negative, females

Fig. 2. A) Spinal deformity index (SDI) and B) kyphosis Z-scores in 18 WNT1 p.C218G mutation-positive and 14 mutation-negative subjects 


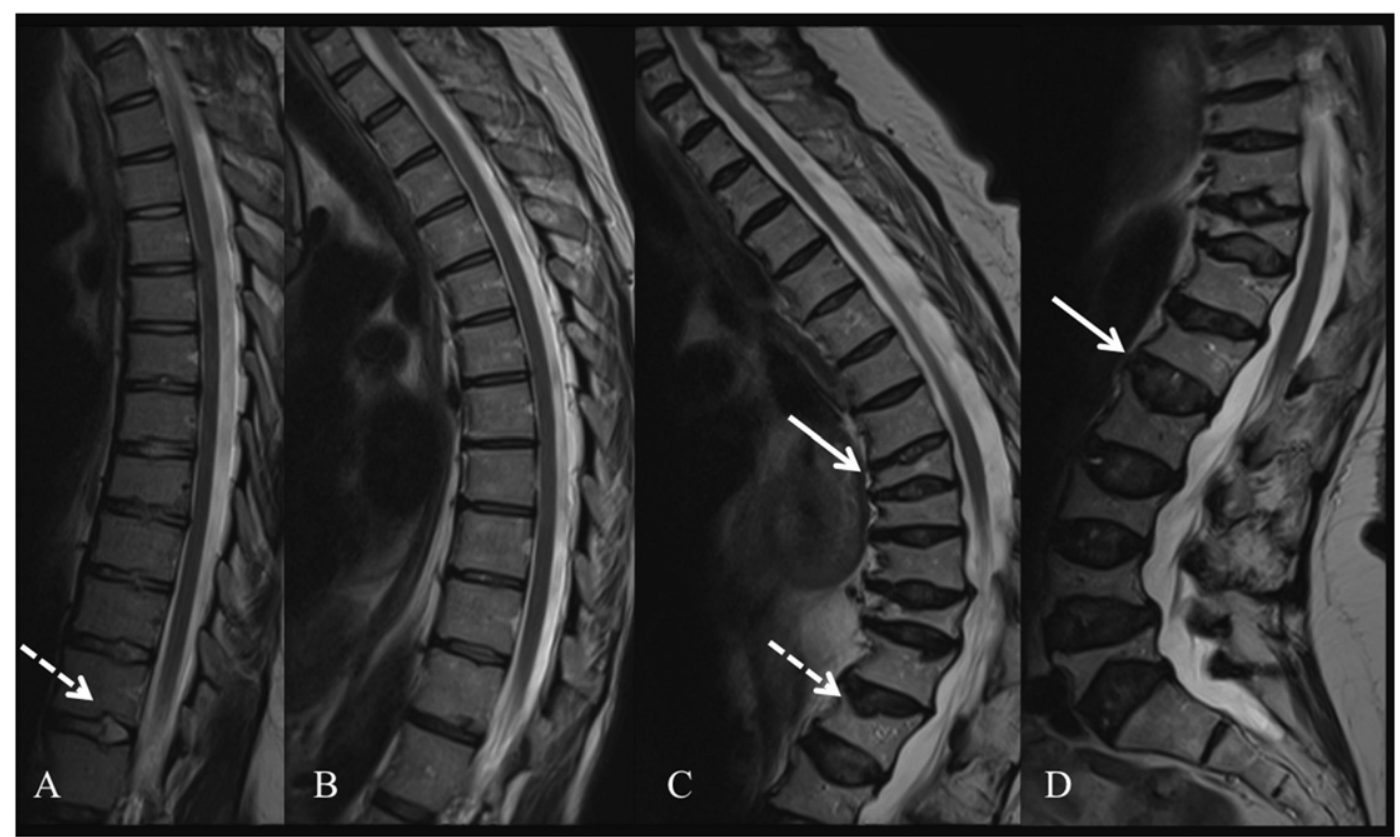

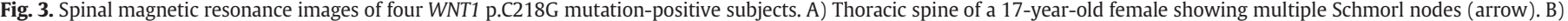

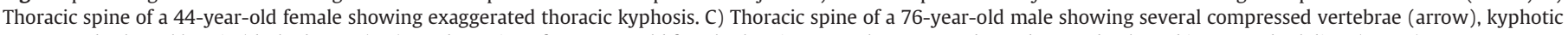
stature and Schmorl hernia (dashed arrow). D) Lumbar spine of a 74-year-old female showing several compressed vertebrae and enlarged intervertebral discs (arrow).

for subjects over the age of 50 years $57^{\circ}$ and $31^{\circ}(p=0.01)$, respectively. When the degree of kyphosis was compared with published age- and sex-specific normative data, there was a significant difference in kyphosis $Z$-scores between the mutation-positive and -negative subjects (Fig. 2B), especially after age 50 years. Thoracic kyphosis was significantly ( $\mathrm{p}=0.02$ ) increased in the mutation-positive subjects; 6 out of 18 mutation-positive subjects had a kyphosis Z-score $>+2.0$ while all mutation-negative subjects had a Z-score within the -1.5 to +1.5 range. There was also an increase in kyphosis with increasing age (Fig. 3A-C). The median values for lumbar lordosis were $41^{\circ}$ and $44^{\circ}$ in the mutation-positive and -negative subjects, respectively $(\mathrm{p}=$ 0.47 ); for subjects under the age of 50 years the values were $35^{\circ}$ and $40^{\circ}(\mathrm{p}=0.40)$ and for those over the age of 50 years, $48^{\circ}$ and $54^{\circ}(\mathrm{p}=$ 0.32 ), respectively.

\section{Discussion}

This study reports for the first time a systematic evaluation of the spine by MRI in a large cohort of patients with an inherited defect in WNT signaling. We assessed the spine in 18 mutation-positive subjects in two families with autosomal dominant WNT1 osteoporosis and in 14 mutation-negative relatives from the same two families. We previously showed that this WNT1 missense mutation p.C218G impairs WNT signaling [15] and that affected individuals have increased peripheral fractures and compromised bone mass accrual in childhood and early-onset osteoporosis in adulthood [20]. Findings of the present study, involving pediatric and adult subjects, suggest that the negative effects of impaired WNT signaling on the spine accumulate with age and lead to significant spinal pathology by mid adulthood. Comprehensive evaluation

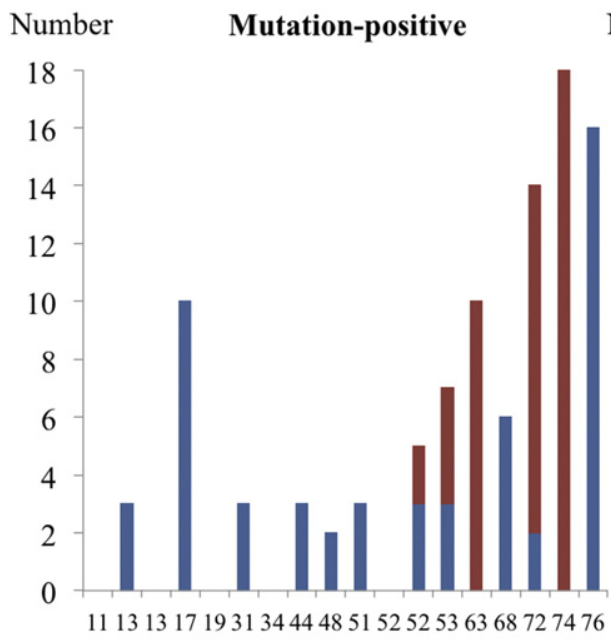

Age (years)
Number Mutation-negative

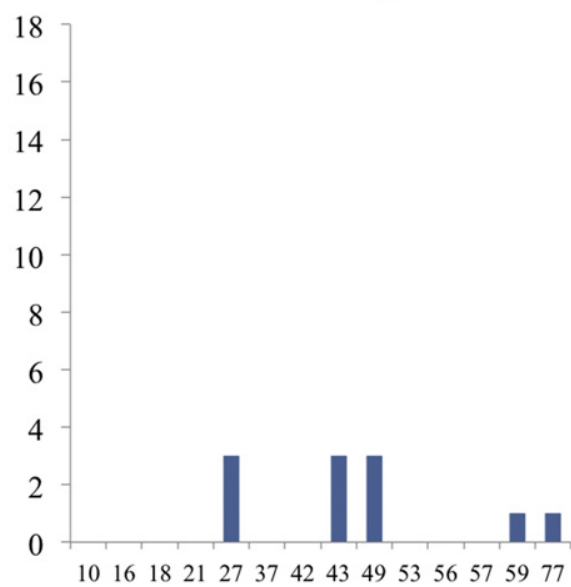

Age (years)

Unassessable endplates 
of the spine, including vertebrae, cartilaginous tissue, and intervertebral discs, by MRI showed that all these spinal structures are affected.

VCFs were common and caused significantly increased kyphosis in mutation-positive adults. All subjects with fractures were older than 50 years, fracture prevalence being $78 \%$ in that age group. Fractures were also more severe and involved more vertebrae than in the mutation-negative subjects (mean SDI $4 \pm 7.3$ vs $0 \pm 0.4$ ). Further, the WNT1 mutation-positive subjects had more often increased thoracic kyphosis (33\% vs $0 \%$ ) and SNs (61\% vs 36\%) than the mutation-negative subjects.

The wide age distribution in our cohort enabled us to make some observations about the vertebral changes' relationship to age. We have previously shown that some skeletal changes are already seen in early childhood and that adults present with severe early-onset osteoporosis $[4,18]$. The present study showed phenotypic variability among subjects with same age. Most of the spinal changes occurred only past the age of 50 years, when a notable increase in pathological findings was seen in both males and females. Similarly, functionally significant LRP5 polymorphisms modulate bone mass accrual during childhood and lead to subnormal lumbar spine BMD by adulthood $[3,27,28]$. In 10 individuals with defective WNT signaling due to a heterozygous LRP5 mutation we showed that the magnitude of BMD deterioration and presence of VCFs associated with the patient's age. It seems likely that the changes in the axial spine accumulate during childhood and young adulthood and when a certain threshold BMD is reached, spinal changes (i.e. compression fractures and kyphosis) occur.

WNT signaling is also of key importance in cartilaginous tissues. WNTs function as a molecular switch in determining mesenchymal stem cells' commitment to either osteoblastic or chondrogenic lineage, and orchestrate their sequential proliferation and differentiation [29]. In the present study, spinal cartilaginous tissue was studied by evaluating defects in spinal endplates and intervertebral discs. SNs were twice as common in the mutation-positive subjects (69\% vs 36\%), were present at all ages and were atypically more frequent in the thoracic spine. They were also more common at an earlier age (one subject had 10 SNs at 17 years). SNs are intravertebral disc herniations often associated with disc degeneration and weakened vertebral bone, and usually seen near the thoracolumbar junction [30]. While our results suggest low BMD to be a risk factor for SNs, the WNT1 mutation may also hamper normal formation of cartilage, making the end plates more prone to herniation. As the SNs were more frequent and had earlier onset in the mutation-positive subjects, the presence of SN may be predictive of future VCFs. This however needs to be evaluated in a longitudinal study. Further, the overall role of WNT signaling in cartilage needs to be addressed in future studies.

Our study has some limitations, mainly concerning the relatively small cohort size and the cross-sectional nature of the study. Also, some of the mutation-positive subjects had received osteoporosis treatment prior to the study, influencing the natural course and improving the skeletal pathology. Importantly however, the presence of significant spinal pathology despite several years' medical treatment suggests that the available treatment modalities are not optimal. We did not use validated methods to evaluate the degree and actual cause of back pain, and physical and quality-of-life consequences of spinal changes; these would be important to gain more understanding of the disease burden. The MRI images were taken in supine position and compared against data taken in standing position. Several studies have indicated that kyphosis is decreased in supine versus standing position [31] and hence our study may have underestimated the degree of kyphosis. Lastly, as our study focuses solely on the spine, no other parts of the skeleton (e.g. knee or hip joints) were included. Evaluation of the appendicular skeleton in future studies will provide more comprehensive understanding of the skeletal and cartilaginous effects of the WNT1 mutation. Despite these limitations, our study provides new and important information regarding the clinical implications of impaired WNT signaling on the spine. This is the largest reported genetically homogeneous cohort of subjects with a mutation in the WNT signaling pathway. The presence of an identical mutation in all affected subjects of all ages, and inclusion of mutation-negative subjects from only two families allowed us to make several observations regarding the spinal changes' relationship to aging.

We conclude that defective WNT signaling due to the WNT1 mutation p.C218G causes significant changes in the spine that are present both in bony vertebrae and in cartilaginous tissues, are evident in almost all subjects over the age of 50 years and become increasingly severe with age. VCFs and exaggerated kyphosis are a clinically significant and common problem, causing pain, physical limitations, impaired quality of life and increased morbidity [32]. Whether these changes are apparent in other tissues of the appendicular skeleton is left unexplored and will be subject to future studies. Therapies targeting the WNT pathway may be an effective way to prevent spinal pathology not only in those harboring a mutation but also in the general population with similar pathology.

\section{Funding}

This study was supported by the Sigrid Jusélius Foundation, the Folkhälsan Research Foundation, the Academy of Finland (277843), the Foundation for Pediatric Research, the Helsinki University Research Funds, the Swedish Research Council (2013-2603), the Novo Nordisk Foundation, the European Society for Paediatric Endocrinology Research Unit, the Swedish Childhood Cancer Foundation, and through the regional agreement on medical training and clinical research (ALF) between Stockholm County Council and Karolinska Institutet. The funders had no role in design and conduct of the study; collection, management, analysis, and interpretation of the data; and preparation, review, or approval of the manuscript.

\section{Authors' roles}

Study design: REM, TN and OM. Study conduct: REM, TN, JN and OM. Data collection: REM, TN, MN, CSJ. Data analysis: REM, TN and JN. Drafting manuscript: REM, TN and OM. Revising manuscript content: all authors. Approving final version of manuscript: all authors. REM and $\mathrm{OM}$ take responsibility for the integrity of the data.

\section{Conflicts of interest}

None.

\section{Acknowledgements}

We thank Hanna Hellsten for help with laboratory work and Esa Liukkonen, Eveliina Lammentausta, Päivi Turunen and Eira Leinonen for help with collecting subject data and samples.

\section{References}

[1] R. Baron, M. Kneissel, WNT signaling in bone homeostasis and disease: from human mutations to treatments, Nat. Med. 19 (2013) 179-192.

[2] Y. Gong, R.B. Slee, N. Fukai, G. Rawadi, S. Roman-Roman, A.M. Reginato, et al., Osteoporosis-pseudoglioma Syndrome Collaborative Group. LDL receptor-related protein 5 (LRP5) affects bone accrual and eye development, Cell 107 (2001) 513-523.

[3] A. Saarinen, T. Saukkonen, T. Kivelä, U. Lahtinen, C. Laine, M. Somer, et al., Low density lipoprotein receptor-related protein 5 (LRP5) mutations and osteoporosis, impaired glucose metabolism and hypercholesterolaemia, Clin. Endocrinol. 72 (2010) 481-488.

[4] C.M. Laine, B.D. Chung, M. Susic, T. Prescott, O. Semler, T. Fiskerstrand, et al., Novel mutations affecting LRP5 splicing in patients with osteoporosis-pseudoglioma syndrome (OPPG), Eur. J. Hum. Genet. 19 (2011) 875-881.

[5] G.G. Loots, M. Kneissel, H. Keller, M. Baptist, J. Chang, N.M. Collette, et al., Genomic deletion of a long-range bone enhancer misregulates sclerostin in Van Buchem disease, Genome Res. 15 (2005) 928-935.

[6] W. Balemans, M. Ebeling, N. Patel, E. Van Hul, P. Olson, M. Dioszegi, et al., Increased bone density in sclerosteosis is due to the deficiency of a novel secreted protein (SOST), Hum. Mol. Genet. 10 (2001) 537-543. 
[7] J.B. van Meurs, T.A. Trikalinos, S.H. Ralston, S. Balcells, M.L. Brandi, K. Brixen, et al, Large-scale analysis of association between LRP5 and LRP6 variants and osteoporosis, JAMA 299 (2008) 1277-1290.

[8] H.F. Zheng, V. Forgetta, Y.H. Hsu, K. Estrada, A. Rosello-Diez, P.J. Leo, et al., Whole-genome sequencing identifies EN1 as a determinant of bone density and fracture, Nature 526 (2015) 112-117.

[9] J.B. Regard, Z. Zhong, B.O. Williams, Y. Yang, Wnt signaling in bone development and disease: making stronger bone with Wnts, Cold Spring Harb. Perspect. Biol. 4 (2012).

[10] T.F. Day, X. Guo, L. Garrett-Beal, Y. Yang, Wnt/beta-catenin signaling in mesenchymal progenitors controls osteoblast and chondrocyte differentiation during vertebrate skeletogenesis, Dev. Cell 8 (2005) 739-750.

[11] M. Almeida, L. Han, T. Bellido, S.C. Manolagas, S. Kousteni, Wnt proteins prevent apoptosis of both uncommitted osteoblast progenitors and differentiated osteoblasts by beta-catenin-dependent and -independent signaling cascades involving $\mathrm{Src}$ ERK and phosphatidylinositol 3-kinase/AKT, J. Biol. Chem. 280 (2005) 41342-41351.

[12] I. Kramer, C. Halleux, H. Keller, M. Pegurri, J.H. Gooi, P.B. Weber, et al., Osteocyte Wnt/beta-catenin signaling is required for normal bone homeostasis, Mol. Cell. Biol. 30 (2010) 3071-3085.

[13] D.A. Glass 2nd, P. Bialek, J.D. Ahn, M. Starbuck, M.S. Patel, H. Clevers, et al., Canonica Wnt signaling in differentiated osteoblasts controls osteoclast differentiation, Dev. Cell 8 (2005) 751-764.

[14] X. Li, M.S. Ominsky, Q.T. Niu, N. Sun, B. Daugherty, D. D'Agostin, et al., Targeted deletion of the sclerostin gene in mice results in increased bone formation and bone strength, J. Bone Miner. Res. 23 (2008) 860-869.

[15] C.M. Laine, K.S. Joeng, P.M. Campeau, R. Kiviranta, K. Tarkkonen, M. Grover, et al, WNT1 mutations in early-onset osteoporosis and osteogenesis imperfecta, N. Engl. J. Med. 368 (2013) 1809-1816.

[16] F.P. Luyten, P. Tylzanowski, R.J. Lories, Wnt signaling and osteoarthritis, Bone 44 (2009) 522-527.

[17] Y. Usami, A.T. Gunawardena, M. Iwamoto, M. Enomoto-Iwamoto, Wnt signaling in cartilage development and diseases: lessons from animal studies, Lab. Investig. 96 (2016) 186-196.

[18] K. Keupp, F. Beleggia, H. Kayserili, A.M. Barnes, M. Steiner, O. Semler, et al., Mutations in WNT1 cause different forms of bone fragility, Am. J. Hum. Genet. 92 (2013) 565-574

[19] S.M. Pyott, T.T. Tran, D.F. Leistritz, M.G. Pepin, N.J. Mendelsohn, R.T. Temme, et al, WNT1 mutations in families affected by moderately severe and progressive recessive osteogenesis imperfecta, Am. J. Hum. Genet. 92 (2013) 590-597.
[20] R.E Mäkitie, M. Haanpää, H. Valta, M. Pekkinen, C.M. Laine, A.E. Lehesjoki, et al. Skeletal characteristics of WNT1 osteoporosis in children and young adults, J. Bone Miner. Res. 31 (2016) 1734-1742.

[21] H.K. Genant, C.Y. Wu, C. Van Kujik, M.C. Nevitt, Vertebral fracture assessment using a semiquantitative technique, J. Bone Miner. Res. 8 (1993) 1137-1148.

[22] H.W. Minne, G. Leidig, C. Wüster, L. Siromachkostov, G. Baldauf, R. Bickel, et al., A newly developed spine deformity index (SDI) to quantitate vertebral crush fractures in patients with osteoporosis, Bone Miner. 3 (1988) 335-349.

[23] S. Kerkeni, S. Kolta, J. Fechtenbaum, C. Roux, Spinal deformity index (SDI) is a good predictor of incident vertebral fractures, Osteoporos. Int. 20 (2009) 1547-1552.

[24] D. Samartzis, F.P. Mok, J. Karppinen, D.Y. Fong, K.D. Luk, K.M. Cheung, Classification of Schmorl's nodes of the lumbar spine and association with disc degeneration: a large-scale population-based MRI study, Osteoarthr. Cartil. 24 (2016) 1753-1760.

[25] J.R. Cobb, Outline for the study of scoliosis, J. Am. Acad. Orthop. Surg. 5 (1948) 261-275.

[26] G.T. Fon, M.J. Pitt, A.C. Thies Jr., Thoracic kyphosis: range in normal subjects, AJR Am. J. Roentgenol. 134 (1980) 979-983.

[27] H. Hartikka, O. Mäkitie, M. Männikkö, A.S. Doria, A. Daneman, W.G. Cole, et al., Heterozygous mutations in the LDL receptor-related protein 5 (LRP5) gene are associated with primary osteoporosis in children, J. Bone Miner. Res. 20 (2005) 783-789.

[28] A. Saarinen, V.V. Välimäki, M.J. Välimäki, E. Löyttyniemi, K. Auro, P. Uusen, et al., The A1330V polymorphism of the low-density lipoprotein receptor-related protein 5 gene (LRP5) associates with low peak bone mass in young healthy men, Bone 40 (2007) 1006-1012.

[29] T.P. Hill, D. Später, M.M. Taketo, W. Birchmeier, C. Hartmann, Canonical Wnt/betacatenin signaling prevents osteoblasts from differentiating into chondrocytes, Dev. Cell 8 (2005) 727-738.

[30] F.M. Williams, N.J. Manek, P.N. Sambrook, T.D. Spector, A.J. Macgregor, Schmorl's nodes: common, highly heritable, and related to lumbar disc disease, Arthritis Rheum. 57 (2007) 855-860.

[31] Y. Hirabayashi, R. Shimizu, K. Saitoh, H. Fukuda, T. Igarashi, M. Furuse, Anatomical configuration of the spinal column in the supine position. III. Comparison of adolescent and adult volunteers, Br. J. Anaesth. 76 (1996) 508-510.

[32] R. Hasserius, M.K. Karlsson, B. Jónsson, I. Redlund-Johnell, O. Johnell, Long-term morbidity and mortality after a clinically diagnosed vertebral fracture in the elderly-a 12- and 22-year follow-up of 257 patients, Calcif. Tissue Int. 76 (2005) 235-242. 